\title{
Quantum fluctuations of voltage in superconducting nanowires
}

\author{
Andrew G. Semenov ${ }^{1,3}$ and Andrei D. Zaikin ${ }^{2,1}$ \\ ${ }^{1}$ I.E. Tamm Department of Theoretical Physics, P.N. Lebedev Physical Institute, Moscow 119991, Russia \\ ${ }^{2}$ Institute of Nanotechnology, Karlsruhe Institute of Technology (KIT), Karlsruhe 76021, Germany \\ E-mail: andrei.zaikin@kit.edu \\ ${ }^{3}$ National Research University Higher School of Economics, Moscow 101000, Russia
}

Received February 2, 2017, published online May 25, 2017

\begin{abstract}
At low temperatures non-equilibrium voltage fluctuations can be generated in current-biased superconducting nanowires due to proliferation of quantum phase slips (QPS) or, equivalently, due to quantum tunneling of magnetic flux quanta across the wire. In this paper we review and further extend recent theoretical results related to this phenomenon. Employing the phase-charge duality arguments combined with Keldysh path integral technique we analyze such fluctuations within the two-point and four-point measurement schemes demonstrating that voltage noise detected in such nanowires in general depends on the particular measurement setup. In the low frequency limit we evaluate all cumulants of the voltage operator which turn out to obey Poisson statistics and exhibit a power law dependence on the external bias. We also specifically address a non-trivial frequency dependence of quantum shot noise power spectrum $S_{\Omega}$ for both longer and shorter superconducting nanowires. In particular, we demonstrate that $S_{\Omega}$ decreases with increasing frequency $\Omega$ and vanishes beyond a threshold value of $\Omega$ at $T \rightarrow 0$. Furthermore, we predict that $S_{\Omega}$ may depend non-monotonously on temperature due to quantum coherent nature of QPS noise. The results of our theoretical analysis can be directly tested in future experiments with superconducting nanowires.
\end{abstract}

PACS: 73.23.Ra Persistent currents;

74.25.F- Transport properties;

74.40.-n Fluctuation phenomena.

Keywords: quantum phase slips and shot noise

\section{Introduction}

Perhaps the most fundamental property of any bulk superconducting material is its ability to conduct electric current without any resistance, i.e., a non-dissipative current below some critical value can pass through such materials. It is clear that in this case neither non-zero average voltage nor voltage fluctuations across the superconductor can be expected. While this simple physical picture holds for sufficiently large superconducting samples (usually well described by means of the standard mean field theory approach), it may change drastically as soon as superconductor dimensions become sufficiently small. In this case thermal and/or quantum fluctuations start playing an important role and the system properties may qualitatively differ from those of bulk superconducting structures. For instance, in the case of ultrathin superconducting wires such fluctuations are responsible for temporal local suppression of the superconducting order parameter $\Delta=|\Delta| \mathrm{e}^{i \varphi}$ inside the wire and, hence, for the phase slippage process. This process gives rise to interesting physical phenomena which cannot be captured with the aid of the mean field theory.

In the low temperature limit thermal fluctuations are unimportant and the system behavior is essentially determined by quantum phase slips (QPS) [1-4]. Each QPS event implies the net phase jump by $\delta \varphi= \pm 2 \pi$ accompanied by a voltage pulse $\delta V=\dot{\varphi} / 2 e$ as well as tunneling of one magnetic flux quantum $\Phi_{0} \equiv \pi / e=\int|\delta V(t)| d t$ across the wire normally to its axis (here and below we set $\hbar=1$ ).

Formally different QPS events can be considered as logarithmically interacting quantum particles [5] forming a 2D gas in space-time characterized by an effective fugacity proportional to the QPS tunneling amplitude per unit wire length [6]

$$
\gamma_{Q P S} \sim\left(g_{\xi} \Delta_{0} / \xi\right) \exp \left(-a g_{\xi}\right), \quad a \sim 1 .
$$


Here $\Delta_{0}$ is the mean field superconducting order parameter, $g_{\xi}=2 \pi \sigma_{N} s /\left(e^{2} \xi\right)>>1$ is the dimensionless normal state conductance of the wire segment of length equal to the coherence length $\xi$, s and $\sigma_{N}$ are respectively the wire cross section and its Drude conductance.

At $T \rightarrow 0$ long superconducting wires exhibit a quantum phase transition [5] controlled by the dimensionless parameter $\lambda \propto \sqrt{s}$ which we will specify later. In ultrathin wires with $\lambda<2$ superconductivity is fully suppressed by quantum fluctuations, and such wires may even go insulating at $T \rightarrow 0$. In somewhat thicker wires with $\lambda>2$ quantum fluctuations are not so efficient, the wire resistance $R$ decreases with $T$ and one gets [5]

$$
R=\frac{d\langle\hat{V}\rangle}{d I} \propto \begin{cases}\gamma_{Q P S}^{2} T^{2 \lambda-3}, & T>>\Phi_{0} I, \\ \gamma_{Q P S}^{2} I^{2 \lambda-3}, & T \ll \Phi_{0} I .\end{cases}
$$

Here and below $\langle\hat{V}\rangle$ is the expectation value of the voltage operator across the wire. According to Eq. (2) the wire nonlinear resistance does not vanish down to lowest temperatures, as it was later confirmed in a number of experiments [7-10].

Can one also expect to observe non-vanishing voltage fluctuations in superconducting nanowires? The presence of QPS-induced equilibrium voltage fluctuations in such nanowires can be predicted already making use of the result (2) combined with the fluctuation-dissipation theorem (FDT). The issue of non-equilibrium voltage fluctuations (e.g., shot noise) is somewhat more complicated. At this stage it is worth to remind the reader two key pre-requisites of shot noise: (i) the presence of discrete charge carriers (e.g., electrons) in the system and (ii) scattering of such carriers at disorder. Although discrete charge carriers Cooper pairs - are certainly present in superconducting nanowires, they form a superconducting condensate flowing along the wire without any scattering. For this reason the possibility for shot noise to occur in superconducting nanowires need to be investigated in more details.

In this paper we will review and extend our recent theoretical analysis of QPS-induced voltage fluctuations in ultrathin superconducting wires [11-13]. In particular, we will proceed beyond FDT and demonstrate that quantum phase slips can generate not only equilibrium but also nonequilibrium voltage fluctuations in ultrathin superconducting wires. Such fluctuations are caused by quantum tunneling of magnetic flux quanta $\Phi_{0}$ and - as we will show obey Poisson statistics. In what follows we will mainly focus our attention to QPS-induced shot noise of the voltage in both long and short nanowires within different measurement schemes and identify highly non-trivial dependencies of the noise power spectrum on temperature, frequency and external current.

The structure of the paper is as follows. In Sec. 2 we define the two models to be analyzed here and present a simple operator derivation of the dual Hamiltonian for a superconducting nanowires in the presence of quantum phase slips. In Sec. 3 we outline our real time Keldysh technique based approach that generally allows us to evaluate all cumulants of the voltage operator perturbatively in the QPS amplitude (1). General expressions for the voltage correlators are derived in Sec. 4. In Sec. 5 we illustrate a direct relation between our real time technique and the quasiequilibrium imaginary time (the so-called $\operatorname{Im} F$ ) approach. Our general results for voltage fluctuations (in particular for shot noise) are further analyzed in Sec. 6 in a number of important limits. In Sec. 7 we consider a four-point measurement setup and compare our results derived in this case with those for the twopoint measurement setup discussed in previous sections. The paper is concluded by a brief summary in Sec. 8 .

\section{Basic models and phase-charge duality}

In this paper we will consider two somewhat different setups which allow to experimentally study voltage fluctuations in superconducting nanowires. The first setup is displayed in Fig. 1. This system consists of an ultrathin superconducting wire of length $L$ and cross section $s$. A capacitance $C$ and a shunt resistor $R_{\mathrm{s}}$ are switched in parallel to this wire. The whole system is biased by an external current $I=V_{X} / R_{X}$. The right wire end $(x=L)$ is grounded as shown in the figure (here and below $x$ is the coordinate along the wire ranging from 0 to $L$ ). The voltage $V(t)$ at its left end $x=0$ fluctuates and such fluctuations can be measured by a detector.

Another possible setup is shown in Fig. 2. It consists of a superconducting nanowire attached to a current source $I$ and two voltage probes located in the points $x_{1}$ and $x_{2}$. The wire contains a thinner segment of length $L$ where quantum phase slips can occur with the amplitude (1).

Both structures displayed in Figs. 1 and 2 can be treated within the same formalism which we are going to outline below. The system shown in Fig. 2 will be addressed below in Sec. 7 of this paper. Here we stick to the system of Fig. 1. An effective Hamiltonian for this system can be expressed in the form

$$
\hat{H}=\hat{H}_{\mathrm{ch}}+\hat{H}_{\text {dis }}-I \varphi / 2 e+\hat{H}_{\text {wire }} .
$$

The first three terms in the right-hand side of Eq. (3) define respectively the charging energy [14],

$$
\hat{H}_{\mathrm{ch}}=\frac{1}{2 C}\left(-i \frac{\partial}{\partial(\varphi / 2 e)}+Q\right)^{2},
$$

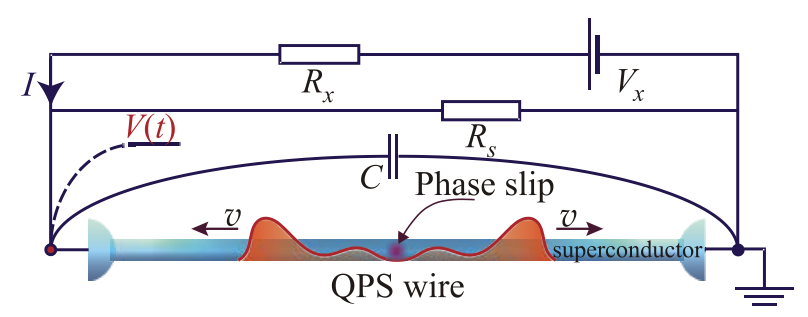

Fig. 1. (Color online) The first setup under consideration. The figure also illustrates creation of two plasmons by a QPS. 


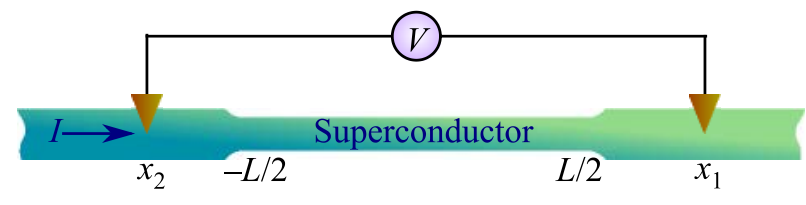

Fig. 2. (Color online) The second setup to be analyzed in Sec. 7.

the Caldeira-Leggett contribution of the shunt resistor $R_{S}$ [14] and the energy tilt produced by an external current $I$. The variable $\varphi(t) \equiv \varphi(0, t)$ denotes the phase of the superconducting order parameter field $\Delta(x, t)$ at $x=0$. Here we also define $\varphi(L, t) \equiv 0$.

The last term $\hat{H}_{\text {wire }}$ in Eq. (3) accounts for the superconducting wire. This part of the effective Hamiltonian can be expressed in terms of both the modulus $|\Delta(x, t)|$ and the phase $\varphi(x, t)$ of the order parameter field [5,6,15]. Here, however, it will be convenient for us to proceed differently and to employ the duality arguments.

The duality between the phase and the charge variables was discussed in details in the case of ultrasmall Josephson junctions [14,16-18]. Later the same duality arguments were extended to short [19] and long [20-22] superconducting wires. Below we will illustrate the formal path integral results [22] by means of a simple operator analysis.

In the absence of quantum phase slips an effective low energy Hamiltonian for a superconducting nanowire can be written in the form

$$
\hat{H}_{\mathrm{eff}}=\int_{0}^{L} d x\left[\frac{\hat{Q}^{2}(x)}{2 C_{w}}+\frac{1}{2 \mathcal{L}_{\mathrm{kin}}}\left(\frac{\partial_{x} \hat{\varphi}(x)}{2 e}\right)^{2}\right],
$$

where $C_{w}$ and $\mathcal{L}_{\text {kin }}=1 /\left(\pi \sigma_{N} \Delta_{0} s\right)$ are respectively the geometric wire capacitance (per length) and the kinetic wire inductance (times length), $\hat{Q}(x)$ and $\hat{\varphi}(x)$ are canonically conjugate local charge and phase operators obeying the commutation relations

$$
\left[\hat{Q}(x), \hat{\varphi}\left(x^{\prime}\right)\right]=-2 i e \delta\left(x-x^{\prime}\right),
$$

As the contribution of the external current source $I$ is already accounted for in Eq. (3), for the sake of our derivation and without loss of generality we can now assume that the superconducting wire is isolated from any external circuit. Then the current at its end points $x=0$ and $x=L$ vanishes and, hence, we can define the boundary conditions for the phase in the form

$$
\partial_{x} \hat{\varphi}(0)=\partial_{x} \hat{\varphi}(L)=0 .
$$

Employing the Fourier series expansion, we get

$$
\begin{aligned}
& \hat{\varphi}(x)=\hat{\varphi}_{0}+\sqrt{\frac{2}{L}} \sum_{n=1}^{\infty} \hat{\varphi}_{n} \cos (\pi n x / L), \\
& \hat{Q}(x)=\frac{\hat{Q}_{0}}{L}+\sqrt{\frac{2}{L}} \sum_{n=1}^{\infty} \hat{Q}_{n} \cos (\pi n x / L),
\end{aligned}
$$

where

$$
\left[\hat{Q}_{0}, \hat{\varphi}_{0}\right]=-2 i e, \quad\left[\hat{Q}_{m}, \hat{\varphi}_{n}\right]=-2 i e \delta_{m n}
$$

Let us now perform the dual transformation. For this purpose we introduce the following (dual) operators

$$
\hat{\Phi}(x)=\partial_{x} \hat{\varphi}(x) / 2 e
$$

and

$$
\hat{\chi}(x)=-\frac{\pi}{e} \int_{x}^{L} d x^{\prime} \hat{Q}\left(x^{\prime}\right)+\frac{\pi(L-x)}{e L} \int_{0}^{L} d x^{\prime} \hat{Q}\left(x^{\prime}\right),
$$

which can also be expressed as

$$
\begin{gathered}
\hat{\Phi}(x)=-\sqrt{\frac{\pi^{2}}{2 e^{2} L^{3}}} \sum_{n=1}^{\infty} n \hat{\varphi}_{n} \sin (\pi n x / L), \\
\hat{\chi}(x)=\sqrt{\frac{2 L}{e^{2}}} \sum_{n=1}^{\infty} \frac{\hat{Q}_{n}}{n} \sin (\pi n x / L) .
\end{gathered}
$$

These new canonically conjugate flux and charge operators obey the commutation relations

$$
\left[\hat{\Phi}(x), \hat{\chi}\left(x^{\prime}\right)\right]=-i \Phi_{0} \delta\left(x-x^{\prime}\right)
$$

and obvious boundary conditions

$$
\hat{\Phi}(0)=\hat{\Phi}(L)=0, \quad \hat{\chi}(0)=\hat{\chi}(L)=0 .
$$

Substituting the relations

$$
\partial_{x} \hat{\varphi}(x)=2 e \hat{\Phi}(x), \quad \hat{Q}(x)=\frac{\hat{Q}_{0}}{L}+\frac{e}{\pi} \partial_{x} \hat{\chi}(x)
$$

into Eq. (5), we obtain

$$
\hat{H}_{\mathrm{eff}}=\frac{\hat{Q}_{0}^{2}}{2 L C_{w}}+\hat{H}_{T L},
$$

where

$$
\hat{H}_{T L}=\int_{0}^{L} d x\left(\frac{\hat{\Phi}^{2}}{2 \mathcal{L}_{\mathrm{kin}}}+\frac{\left(\partial_{x} \hat{\chi}\right)^{2}}{2 C_{w} \Phi_{0}^{2}}\right)
$$

is the Hamiltonian for a transmission line formed by a superconducting wire.

The physical meaning of the operator $\hat{\chi}(x, t)$ is transparent: It is simply proportional to the operator for the charge that has passed through the point $x$ up to the time moment $t$. Hence, the local current and the local charge density operators are defined respectively as

$$
\hat{I}(x, t)=\partial_{t} \hat{\chi}(x, t) / \Phi_{0}, \quad \hat{\rho}(x, t)=-\partial_{x} \hat{\chi}(x, t) / \Phi_{0} .
$$

The charge $Q$ in Eq. (4) equals to $Q(t)=\chi(0, t) / \Phi_{0}$.

The above consideration does not yet account for quantum phase slips. In order to specify the QPS contribution to 
the wire Hamiltonian let us first define the phase field configurations as

$$
\hat{\varphi}(x)|\varphi(x)\rangle=\varphi(x)|\varphi(x)\rangle
$$

and bear in mind that the phase of the superconducting order parameter is a compact variable. Accordingly, the field configurations $\varphi(x)$ and $\varphi(x)+2 \pi$ correspond to the same quantum state of our system. Furthermore, in the absence of QPS, i.e., provided the absolute value of the order parameter does not fluctuate $|\Delta(x, t)|=\Delta_{0}$, also the states $\varphi$ and $\tilde{\varphi}(x)=\varphi(x)+2 \pi \theta\left(x-x_{1}\right)$ (where $0<x_{1}<L$ and $\theta(x)$ is the standard Heaviside step function equal to 0 for $x \leq 0$ and to 1 for $x>0$ ) are physically indistinguishable. For instance, the supercurrent operator proportional to the combination $\Delta_{0}^{2} \exp (-i \hat{\varphi}(x)) \partial_{x} \exp (i \hat{\varphi}(x))$ remains the same in both cases.

Let us now make the step function continuous by effectively smearing it at the scale of the superconducting coherence length $\xi$, i.e., we substitute $\theta(x) \rightarrow \theta_{\xi}(x)$. The corresponding field configuration $\tilde{\varphi}_{\xi}(x)=\varphi(x)+2 \pi \theta_{\xi}\left(x-x_{1}\right)$, on one hand, remains very close to $\tilde{\varphi}(x)$ and, on the other hand, is already physically distinguishable from the latter. The QPS process can be viewed as quantum tunneling between these two close but physically different phase configurations.

What remains is to make use of the fact that the wire Hamiltonian does not depend on the operator $\hat{\varphi}_{0}$, implying that any shift by a constant phase does not change the state of our system. Hence, without loss of generality we can set $\hat{\varphi}_{0}|\psi\rangle=0$ for any system state $|\psi\rangle$. This condition applies for the evolution controlled by the Hamiltonian (5) and it is also maintained in the presence of quantum phase slips. With this in mind we conclude that the QPS process corresponds to quantum tunneling of the phase between the states $\varphi(x)$ and

$$
\varphi^{\prime}(x)=\varphi(x)+2 \pi \theta_{\xi}\left(x-x_{1}\right)-2 \pi \int_{0}^{L} d x \theta_{\xi}\left(x-x_{1}\right) .
$$

In the operator language this tunneling process can be denoted as $\hat{U}_{\xi}\left(x_{1}\right)|\varphi(x)\rangle=\left|\varphi^{\prime}(x)\right\rangle$, where the expression for $\hat{U}_{\xi}\left(x_{1}\right)$ just follows from the commutation relations and reads

$$
\hat{U}_{\xi}\left(x_{1}\right)=\exp \left(\frac{i \pi}{e} \int_{0}^{L} d x\left(\hat{Q}(x)-\hat{Q}_{0} / L\right) \theta_{\xi}\left(x-x_{1}\right)\right) .
$$

As a result, the part of the Hamiltonian which explicitly accounts for the QPS process takes the form

$$
\begin{gathered}
\hat{H}_{Q P S}=-\int_{0}^{L} d x_{1} \gamma_{Q P S}\left(x_{1}\right) \times \\
\times \cos \left(\frac{\pi}{e} \int_{0}^{L} d x\left(\hat{Q}(x)-\hat{Q}_{0} / L\right) \theta_{\xi}\left(x-x_{1}\right)\right),
\end{gathered}
$$

where $\gamma_{Q P S}\left(x_{1}\right)$ is the QPS amplitude at the wire point $x=x_{1}$. Setting now $\xi \rightarrow 0$ and making use of the second Eq. (17), in the case of a uniform wire with $\gamma_{Q P S}(x)=\gamma_{Q P S}$ we obtain

$$
\hat{H}_{Q P S}=-\gamma_{Q P S} \int_{0}^{L} d x \cos \hat{\chi}
$$

This result completes our derivation of the dual representation for the Hamiltonian of a superconducting nanowire. Note that the first term in the right-hand side of Eq. (18) describes an extra contribution to the system charging energy (4), i.e. this term can simply be eliminated by absorbing the total wire capacitance $L C_{w}$ into $C$ as $C+L C_{w} \rightarrow C$. The dual Hamiltonian of the wire in Eq. (3) is then defined by an effective sine-Gordon model

$$
\hat{H}_{\text {wire }}=\hat{H}_{T L}+\hat{H}_{Q P S} \text {. }
$$

\section{Keldysh perturbation theory and Green functions}

Let us now investigate fluctuations of the voltage $V(t)$ in the presence of quantum phase slips. In order to proceed we will employ the dual Hamiltonian (3) derived in the previous section and make use of the Keldysh path integral technique. As usually, our variables of interest are defined on the forward and backward time branches of the Keldysh contour, i.e. we now have $\varphi_{F, B}(t)$ and $\chi_{F, B}(x, t)$. We also routinely introduce "classical" and "quantum" variables, respectively $\varphi_{+}(t)=\left(\varphi_{F}(t)+\varphi_{B}(t)\right) / 2$ and $\varphi_{-}(t)=\varphi_{F}(t)-\varphi_{B}(t)$ (the same recipe holds for the $\chi$-fields).

Employing the Josephson relation between the voltage and the phase one can formally express the expectation value of the voltage operator across the the superconducting wire in the form

$$
\left\langle V\left(t_{1}\right)\right\rangle=\frac{1}{2 e}\left\langle\dot{\varphi}_{+}\left(t_{1}\right) \mathrm{e}^{i S_{Q P S}}\right\rangle_{0},
$$

where

$$
S_{Q P S}=-2 \gamma_{Q P S} \int d t \int_{0}^{L} d x \sin \left(\chi_{+}\right) \sin \left(\chi_{-} / 2\right)
$$

and

$$
\langle\ldots\rangle_{0}=\int \mathcal{D}^{2} \varphi(t) \mathcal{D}^{2} \chi(x, t)(\ldots) \mathrm{e}^{i S_{0}[\varphi, \chi]}
$$

implies averaging with the Keldysh effective action $S_{0}$ corresponding to the Hamiltonian $\hat{H}_{0}=\hat{H}-\hat{H}_{Q P S}$.

Analogously, for any higher order correlator of voltages we have

$$
\begin{gathered}
\left\langle V\left(t_{1}\right) V\left(t_{2}\right) \ldots V\left(t_{n}\right)\right\rangle=\frac{1}{(2 e)^{n}} \times \\
\times\left\langle\dot{\varphi}_{+}\left(t_{1}\right) \dot{\varphi}_{+}\left(t_{2}\right) \ldots \dot{\varphi}_{+}\left(t_{n}\right) \mathrm{e}^{i S} Q P S\right\rangle_{0},
\end{gathered}
$$


At this stage let us emphasize that Eq. (30) defines the symmetrized voltage correlators. E.g., for $n=2$ we have

$$
\left\langle V\left(t_{1}\right) V\left(t_{2}\right)\right\rangle=\frac{1}{2}\left\langle\hat{V}\left(t_{1}\right) \hat{V}\left(t_{2}\right)+\hat{V}\left(t_{2}\right) \hat{V}\left(t_{1}\right)\right\rangle,
$$

while for $n=3$ one finds [23]

$$
\begin{gathered}
\left\langle V\left(t_{1}\right) V\left(t_{2}\right) V\left(t_{3}\right)\right\rangle=\frac{1}{8}\left\{\left\langle\hat{V}\left(t_{1}\right)\left(\mathcal{T} \hat{V}\left(t_{2}\right) \hat{V}\left(t_{3}\right)\right)\right\rangle+\right. \\
+\left\langle\left(\tilde{\mathcal{T}} \hat{V}\left(t_{2}\right) \hat{V}\left(t_{3}\right)\right) \hat{V}\left(t_{1}\right)\right\rangle+\left\langle\hat{V}\left(t_{2}\right)\left(\mathcal{T} \hat{V}\left(t_{1}\right) \hat{V}\left(t_{3}\right)\right)\right\rangle+ \\
+\left\langle\left(\tilde{\mathcal{T}} \hat{V}\left(t_{1}\right) \hat{V}\left(t_{3}\right)\right) \hat{V}\left(t_{2}\right)\right\rangle+\left\langle\hat{V}\left(t_{3}\right)\left(\mathcal{T} \hat{V}\left(t_{1}\right) \hat{V}\left(t_{2}\right)\right)\right\rangle+ \\
+\left\langle\left(\tilde{\mathcal{T}}\left(t_{1}\right) \hat{V}\left(t_{2}\right)\right) \hat{V}\left(t_{3}\right)\right\rangle+\left\langle\mathcal{T} \hat{V}\left(t_{1}\right) \hat{V}\left(t_{2}\right) \hat{V}\left(t_{3}\right)\right\rangle+ \\
\left.+\left\langle\tilde{\mathcal{T}}\left(t_{1}\right) \hat{V}\left(t_{2}\right) \hat{V}\left(t_{3}\right)\right\rangle\right\},
\end{gathered}
$$

where $\mathcal{T}$ and $\tilde{\mathcal{T}}$ are, respectively, the forward and backward time ordering operators.

A formally exact expression (30) can be evaluated perturbatively in the tunneling amplitude $\gamma_{Q P S}(1)$. In the zero order in $\gamma_{Q P S}$ the problem is described by the quadratic (in both $\varphi$ and $\chi$ ) action $S_{0}$. In that case it is necessary to employ the averages

$$
\begin{aligned}
\left\langle\varphi_{+}(t)\right\rangle_{0}=\left\langle\varphi_{-}(t)\right\rangle_{0} & =\left\langle\chi_{-}(x, t)\right\rangle_{0}=0, \\
\left\langle\chi_{+}(x, t)\right\rangle_{0} & =\Phi_{0} I t,
\end{aligned}
$$

as well as the following Green functions

$$
\begin{gathered}
G_{a b}^{K}\left(X, X^{\prime}\right)=-i\left\langle a_{+}(X) b_{+}\left(X^{\prime}\right)\right\rangle_{0}+i\left\langle a_{+}(X)\right\rangle_{0}\left\langle b_{+}\left(X^{\prime}\right)\right\rangle_{0}, \\
G_{a b}^{R}\left(X, X^{\prime}\right)=-i\left\langle a_{+}(X) b_{-}\left(X^{\prime}\right)\right\rangle_{0}
\end{gathered}
$$

where $a(X)$ and $b(X)$ stand for one of the fields $\varphi(t)$ and $\chi(x, t)$. As these fields are real, the Green functions satisfy the condition $G_{a b}^{A}(\omega)=G_{b a}^{R}(-\omega)$. Then the Keldysh function $G^{K}$ takes the form

$$
G_{a b}^{K}(\omega)=\frac{1}{2} \operatorname{coth}\left(\frac{\omega}{2 T}\right)\left(G_{a b}^{R}(\omega)-G_{b a}^{R}(-\omega)\right) .
$$

Expanding Eq. (30) up to the second order in $\gamma_{Q P S}$ and performing all necessary averages, one can express the results in terms of the Green functions (34). These results can be represented graphically in the form of the so-called candy diagrams [11]. These diagrams for the first and the second moments of the voltage operator are displayed in Fig. 3. They involve four different propagators $\left(G_{\chi \chi}^{R, K}\right.$ and

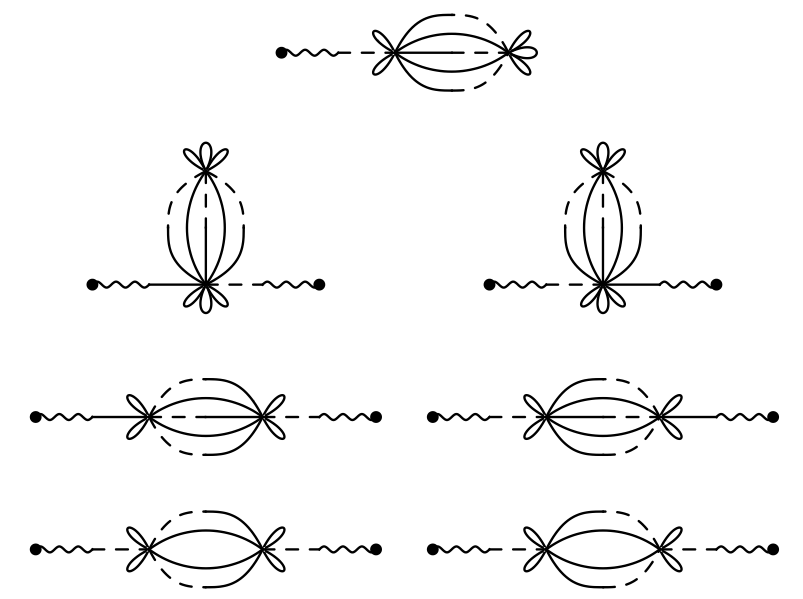

Fig. 3. Candy-like diagrams which determine both average voltage $\langle V\rangle$ (upper diagram) and voltage-voltage corrrelator $\langle V V\rangle$ (six remaining diagrams) in the second order in $\gamma_{Q P S}$. The fields $\varphi_{+}, \chi_{+}$and $\chi_{-}$in the propagators (34) are denoted respectively by wavy, solid and dashed lines.

$G_{\varphi \chi}^{R, K}$ ) and plenty of vertices originating from Taylor expansion of the cosine terms. Summing up all the diagrams in the same order in $\gamma_{Q P S}$ one arrives at the final expression containing the exponents of the Green functions.

What remains is to evaluate all the above Green functions for the system depicted in Fig. 1. This task can be carried out in a straightforward manner. E.g., for the function $G_{\varphi \varphi}^{R}$ we obtain [11]

$$
G_{\varphi \varphi}^{R}(\omega)=\frac{1}{\frac{\omega^{2}}{2 E_{C}}+\frac{i \omega}{4 e^{2} R_{\mathrm{tot}}}-\frac{\omega \lambda}{\pi} \operatorname{coth}\left(\frac{\omega L}{v}\right)},
$$

where $E_{C}=2 e^{2} / C, v=1 / \sqrt{\mathcal{L}_{\text {kin }} C_{w}}$ is the plasmon velocity [24] and the parameter $\lambda$ already introduced above is defined as $\lambda=R_{Q} /\left(2 Z_{w}\right)$ with $R_{Q}=\pi /\left(2 e^{2}\right)$ being the "superconducting" quantum resistance unit and $Z_{w}=\sqrt{\mathcal{L}_{\text {kin }} / C_{w}}$ being the wire impedance. We also defined $R_{\mathrm{tot}}=R_{X} R_{S} /\left(R_{X}+R_{S}\right)$.

The corresponding expressions for $G_{\chi \varphi}^{R}$ and $G_{\chi \chi}^{R}$ read [11]

$$
\begin{gathered}
G_{\chi \varphi}^{R}(x ; \omega)=-G_{\varphi \chi}^{R}(x ; \omega)= \\
=\frac{2 i \lambda \cos \left(\frac{\omega(L-x)}{v}\right)}{\left(\frac{\omega^{2}}{2 E_{C}}+\frac{i \omega}{4 e^{2} R_{\mathrm{tot}}}\right) \sin \left(\frac{\omega L}{v}\right)-\frac{\omega \lambda}{\pi} \cos \left(\frac{\omega L}{v}\right)},
\end{gathered}
$$




$$
\begin{gathered}
G_{\chi \chi}^{R}\left(x, x^{\prime} ; \omega\right)=\frac{1}{\omega \sin \left(\frac{\omega L}{v}\right)} 4 \pi \lambda\left(\cos \left(\frac{\omega(L-x)}{v}\right) \cos \left(\frac{\omega x^{\prime}}{v}\right) \theta\left(x-x^{\prime}\right)+\cos \left(\frac{\omega\left(L-x^{\prime}\right)}{v}\right) \cos \left(\frac{\omega x}{v}\right) \theta\left(x^{\prime}-x\right)\right)+ \\
+\frac{4 \lambda^{2} \cos \left(\frac{\omega(L-x)}{v}\right) \cos \left(\frac{\omega\left(L-x^{\prime}\right)}{v}\right)}{\sin \left(\frac{\omega L}{v}\right)\left(\left(\frac{\omega^{2}}{2 E_{C}}+\frac{i \omega}{4 e^{2} R_{\mathrm{tot}}}\right) \sin \left(\frac{\omega L}{v}\right)-\frac{\omega \lambda}{\pi} \cos \left(\frac{\omega L}{v}\right)\right)}
\end{gathered}
$$

In order to simplify the above expressions let us make use of the momentum conservation for plasmons propagating along the wire. Such plasmons can only be created in pairs with the total zero momentum. Excitations moving towards the grounded end of the superconducting nanowire eventually vanish there with no chance to reappear again while plasmons propagating in the opposite direction produce voltage fluctuations measured by a detector. Then in the long wire limit the general expressions for $G_{\varphi \chi}^{R}$ and $G_{\chi \chi}^{R}$ reduce to more simple ones

$$
\begin{gathered}
G_{\varphi \chi}^{R}(x ; \omega) \simeq-\frac{2 \lambda \mathrm{e}^{i \frac{\omega x}{v}}}{(\omega+i 0)\left(\frac{\omega}{2 E_{C}}+\frac{i \lambda}{\pi}\right)}, \\
G_{\chi \chi}^{R}\left(x, x^{\prime} ; \omega\right) \simeq-\frac{2 \pi i \lambda}{\omega+i 0} \mathrm{e}^{i \frac{\omega\left|x-x^{\prime}\right|}{v}} .
\end{gathered}
$$

In Eqs. (39) and (40) we also set $R_{X}, R_{S} \rightarrow \infty$.

\section{I-V curve and voltage noise: general results}

Making use of the above results it is now straightforward to derive general expressions for the voltage correlators (30). Here we restrict our analysis to the first two moments of the voltage operator. For the expectation value of this operator we obtain

$$
\begin{aligned}
\langle V\rangle= & \frac{i \gamma_{Q P S}^{2}}{4 e} \int_{0}^{L} d x \int_{0}^{L} d x^{\prime}\left(\lim _{\omega \rightarrow 0} \omega G_{\varphi \chi}^{R}(x ; \omega)\right) \times \\
& \times\left(\mathcal{P}_{X, x^{\prime}}\left(-\Phi_{0} I\right)-\mathcal{P}_{X, x^{\prime}}\left(\Phi_{0} I\right)\right),
\end{aligned}
$$

where $\mathcal{P}_{x, x^{\prime}}(\omega)=P_{x, x^{\prime}}(\omega)+\bar{P}_{x, x^{\prime}}(\omega)$ and

$$
\begin{gathered}
P_{x, x^{\prime}}(\omega)=\int_{0}^{\infty} d t \mathrm{e}^{i \omega t} \mathrm{e}^{i \mathcal{G}\left(x, x^{\prime} ; t, 0\right)}, \\
\mathcal{G}\left(x, x^{\prime} ; t, 0\right)=G_{\chi \chi}^{K}\left(x, x^{\prime} ; t, 0\right)-\frac{1}{2} G_{\chi \chi}^{K}(x, x ; t, t)- \\
-\frac{1}{2} G_{\chi \chi}^{K}\left(x^{\prime}, x^{\prime} ; 0,0\right)+\frac{1}{2} G_{\chi \chi}^{R}\left(x, x^{\prime} ; t, 0\right) .
\end{gathered}
$$

With the aid of the identity $\lim _{\omega \rightarrow 0} \omega G_{\varphi \chi}^{R}(x ; \omega)=2 \pi i$ Eq. (41) can be expressed in the form

$$
\langle V\rangle=\Phi_{0}\left(\Gamma_{Q P S}(I)-\Gamma_{Q P S}(-I)\right),
$$

where we defined

$$
\Gamma_{Q P S}(I)=\frac{\gamma_{Q P S}^{2}}{2} \int_{0}^{L} d x \int_{0}^{L} d x^{\prime} \mathcal{P}_{x, x^{\prime}}\left(\Phi_{0} I\right) .
$$

Turning to voltage fluctuations we identify three different contributions to the noise power spectrum

$$
S_{\Omega}=\int d t e^{i \Omega t}\langle V(t) V(0)\rangle=S_{\Omega}^{(0)}+S_{\Omega}^{r}+S_{\Omega}^{a} .
$$

The first of these contributions $S_{\Omega}^{(0)}$ is unrelated to QPS. It just defines equilibrium voltage noise for a transmission line and reads

$$
S_{\Omega}^{(0)}=\frac{i \Omega^{2} \operatorname{coth}\left(\frac{\Omega}{2 T}\right)}{16 e^{2}}\left(G_{\varphi \varphi}^{R}(\Omega)-G_{\varphi \varphi}^{R}(-\Omega)\right) .
$$

The other two terms are due to QPS effects. The term $S_{\Omega}^{r}$ is also proportional to coth $(\Omega / 2 T)$ and depends on the products of two retarded (advanced) Green functions:

$$
\begin{aligned}
& S_{\Omega}^{r}=\frac{\gamma_{Q P S}^{2} \Omega^{2} \operatorname{coth}\left(\frac{\Omega}{2 T}\right)}{8 e^{2}} \int_{0}^{L} d x \int_{0}^{L} d x^{\prime} \operatorname{Re}\left[G_{\varphi \chi}^{R}(x ; \Omega) \times\right. \\
& \left.\quad \times\left(\mathcal{F}_{x, x^{\prime}}(\Omega) G_{\varphi \chi}^{R}\left(x^{\prime} ; \Omega\right)-\mathcal{F}_{x, x^{\prime}}(0) G_{\varphi \chi}^{R}(x ; \Omega)\right)\right] .
\end{aligned}
$$

Here we also denoted

$$
\begin{gathered}
\mathcal{F}_{X, x^{\prime}}(\Omega)=-P_{X, x^{\prime}}\left(\Omega+\Phi_{0} I\right)-P_{X, x^{\prime}}\left(\Omega-\Phi_{0} I\right)+ \\
+\bar{P}_{x, x^{\prime}}\left(-\Omega+\Phi_{0} I\right)+\bar{P}_{x, x^{\prime}}\left(-\Omega-\Phi_{0} I\right) .
\end{gathered}
$$

The remaining term $S_{\Omega}^{a}$, in contrast, contains the product of one retarded and one advanced Green functions. We get

$$
\begin{aligned}
& S_{\Omega}^{a}=\frac{\gamma_{Q P S}^{2} \Omega^{2}}{16 e^{2}} \int_{0}^{L} d x \int_{0}^{L} d x^{\prime} G_{\varphi \chi}^{R}(x ; \Omega) G_{\varphi \chi}^{R}\left(x^{\prime} ;-\Omega\right) \times \\
& \times\left[\sum_{ \pm} \mathcal{C}_{ \pm}\left(\mathcal{P}_{X, x^{\prime}}\left(\Omega \pm \Phi_{0} I\right)-\mathcal{P}_{X, x^{\prime}}\left(-\Omega \mp \Phi_{0} I\right)\right)\right],
\end{aligned}
$$


where

$$
\mathcal{C}_{ \pm}=\operatorname{coth}\left(\frac{\Omega \pm \Phi_{0} I}{2 T}\right)-\operatorname{coth}\left(\frac{\Omega}{2 T}\right)
$$

Eqs. (45)-(50) together with the expressions for the Green functions (36)-(38) fully determine the voltage noise power spectrum of a superconducting nanowire in the perturbative in QPS regime.

\section{Relation to $\operatorname{Im} F$-method}

Comparing Eq. (43) for the average voltage with the corresponding result [5] we can identify the quantity $\Gamma_{\text {QPS }}(I)(44)$ as a quantum decay rate of the current state due to QPS. In [5] this rate was derived with the aid of the so-called $\operatorname{Im} F$-method [25]. It is of interest to establish a direct relation between the latter approach and the real time Keldysh technique employed here.

Let us introduce the generalized Green function $\mathcal{G}_{\chi}\left(x, x^{\prime} ; \sigma\right)$ which depends on the complex time $\sigma$ and satisfies the condition $\mathcal{G}_{\chi}\left(x, x^{\prime} ; t-i 0\right)=\mathcal{G}\left(x, x^{\prime} ; t, 0\right)$ at $t>0$. This function reads

$$
\begin{gathered}
\mathcal{G}_{\chi}\left(x, x^{\prime} ; \sigma\right)=\frac{i T}{2} \int d t \operatorname{coth}(\pi T(t-\sigma)) \times \\
\times \int \frac{d \omega}{2 \pi} \mathrm{e}^{-i \omega t}\left(G_{\chi \chi}^{R}\left(x, x^{\prime} ; \omega\right)-G_{\chi \chi}^{R}\left(x, x^{\prime} ;-\omega\right)\right)
\end{gathered}
$$

The function (51) is analytic, periodic in the imaginary time,

$$
\mathcal{G}_{\chi}\left(x, x^{\prime} ; \sigma\right)=\mathcal{G}_{\chi}\left(x, x^{\prime} ; \sigma-i / T\right)
$$

and has branch cuts at $\operatorname{Im}(\sigma)=N / T$ for all integer $N$. On the imaginary axis the function $\mathcal{G}_{\chi}$ matches with the Matsubara Green function

$$
\mathcal{G}_{\chi}\left(x, x^{\prime} ;-i \tau\right)=i G_{\chi \chi}^{M}\left(x, x^{\prime} ; \tau\right) .
$$

The quantum decay rate $\Gamma$ of a metastable state can be evaluated by means of the well known formula [25]

$$
\Gamma=2 \operatorname{Im} F,
$$

where $F$ is the system free energy. In order to establish the QPS contribution to $\Gamma$ it is necessary to identify the corresponding correction to the free energy $\delta F$. In the leading order in $\gamma_{Q P S}$ one can consider just one QPS-antiQPS pair [5] which yields the following contribution

$$
\delta F=\frac{\gamma_{Q P S}^{2}}{2} \int_{0}^{L} d x \int_{0}^{L} d x^{\prime} \int_{0}^{1 / T} d \tau \mathrm{e}^{-S_{\text {pair }}},
$$

where

$$
S_{\text {pair }}=-\Phi_{0} I \tau+\mathcal{V}\left(x, \tau ; x^{\prime}, 0\right)
$$

and $\tau$ is the imaginary time interval between QPS and antiQPS events. The term $\mathcal{V}\left(x ; x^{\prime} ; \tau, 0\right)$ accounts for the interaction between these events which occur respectively at the points $x$ and $x^{\prime}$. Expressing this interaction term via the Matsubara Green function, we find

$$
\begin{gathered}
\mathcal{V}\left(x ; x^{\prime} ; \tau, 0\right)=G_{\chi \chi}^{M}\left(x, x^{\prime} ; \tau\right)- \\
-\frac{1}{2} G_{\chi \chi}^{M}(x, x ; 0)-\frac{1}{2} G_{\chi \chi}^{M}\left(x^{\prime}, x^{\prime} ; 0\right) .
\end{gathered}
$$

An attentive reader may have already noticed that the integral over $\tau$ in Eq. (55) formally diverges at low temperatures. As a consequence, the free energy acquires an imaginary part $\operatorname{Im} F$ derived with the aid of a proper analytic continuation of $\delta F$. Evaluating the integral (55) by the steepest descent method we routinely determine a stationary point $\tau_{s}$ from the stationary condition for the action

$$
\Phi_{0} I=\partial_{\tau} G_{\chi \chi}^{M}\left(x, x^{\prime} ; \tau_{s}\right) .
$$

A closer inspection allows to conclude that this stationary point delivers a maximum to the action rather than a minimum, thus indicating an instability with respect to quantum decay to lower energy states. In this case the correct recipe is to deform the integration contour along the steepest descent path. This procedure is illustrated in Fig. 4. The initial integration contour goes vertically from 0 to $-i \beta$. This contour can be deformed and directed along the real time axis after passing through the point $\tau_{s}$. Then we obtain

$$
\begin{gathered}
\delta F=\frac{\gamma_{Q P S}^{2}}{2} \int_{0}^{L} d x \int_{0}^{L} d x^{\prime} \int_{0}^{\tau_{S}} d \tau \mathrm{e}^{\Phi_{0} I \tau-\mathcal{V}\left(x ; x^{\prime} ; \tau, 0\right)}+ \\
+\frac{\gamma_{Q P S}^{2}}{2} \int_{0}^{L} d x \int_{0}^{L} d x^{\prime} \int_{0} i d \tau \mathrm{e}^{\Phi_{0} I\left(\tau_{S}+i \tau\right)-\mathcal{V}\left(x ; x^{\prime} ; \tau_{S}+i \tau, 0\right)} .
\end{gathered}
$$

The imaginary part of this expression reads

$$
\begin{aligned}
2 \operatorname{Im} F & =\frac{\gamma_{Q P S}^{2}}{2} \int_{0}^{L} d x \int_{0}^{L} d x^{\prime} \int_{0} d \tau \mathrm{e}^{\Phi_{0} I\left(\tau_{s}+i \tau\right)-\mathcal{V}\left(x ; x^{\prime} ; \tau_{s}+i \tau, 0\right)}+ \\
& +\frac{\gamma_{Q P S}^{2}}{2} \int_{0}^{L} d x \int_{0}^{L} d x^{\prime} \int_{0} d \tau \mathrm{e}^{\Phi_{0} I\left(\tau_{s}-i \tau\right)-\mathcal{V}\left(x ; x^{\prime} ; \tau_{s}-i \tau, 0\right)}
\end{aligned}
$$

Expressing Eq. (60) as a single integral along the contour passing through the point $\tau_{s}$ in the direction perpendicular to real $\tau$ axis, we get

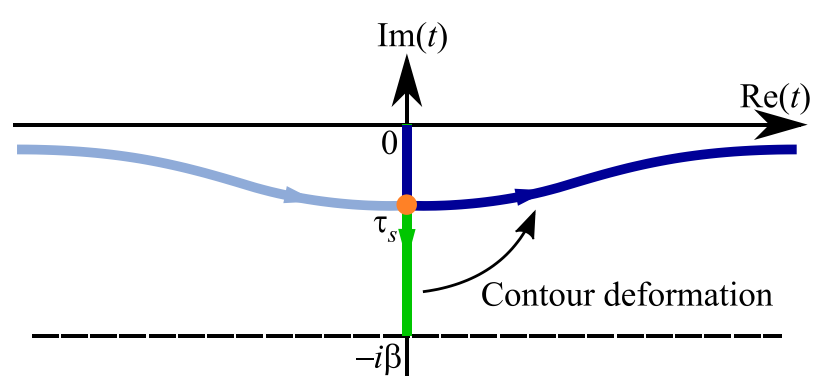

Fig. 4. (Color online) Integration contour. 
$2 \operatorname{Im} F=\frac{\gamma_{Q P S}^{2}}{2} \int_{0}^{L} d x \int_{0}^{L} d x^{\prime} \int d \tau \mathrm{e}^{\Phi_{0} I\left(\tau_{s}+i \tau\right)-\mathcal{V}\left(x ; x^{\prime} ; \tau_{s}+i \tau, 0\right)}$

Combined with Eqs. (57) and (53), this expression can be cast to the form

$$
\begin{aligned}
& 2 \operatorname{Im} F=\frac{\gamma_{Q P S}^{2}}{2} \int_{0}^{L} d x \int_{0}^{L} d x^{\prime} \int d t \mathrm{e}^{\Phi_{0} I\left(\tau_{s}+i t\right)} \times
\end{aligned}
$$

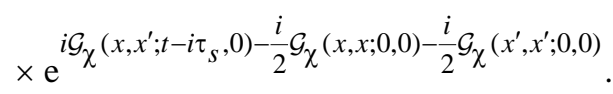

Then making use of the relation

$$
\mathcal{P}_{x, x^{\prime}}(\omega)=\int_{-\infty}^{\infty} d t \mathrm{e}^{i \omega t} \mathrm{e}^{i \mathcal{G}_{\chi}\left(x, x^{\prime} ; t-i 0\right)-\frac{i}{2} \mathcal{G}_{\chi}(x, x ; 0,0)-\frac{i}{2} \mathcal{G}_{\chi}\left(x^{\prime}, x^{\prime} ; 0,0\right)}
$$

we arrive at the final result

$$
2 \operatorname{Im} F=\frac{\gamma_{Q P S}^{2}}{2} \int_{0}^{L} d x \int_{0}^{L} d x^{\prime} \mathcal{P}_{X, x^{\prime}}\left(\Phi_{0} I\right)
$$

This expression together with Eq. (54) confirms that Eq. (44) indeed determines the QPS-mediated decay rate of the current states in a superconducting nanowire, thus proving the equivalence of the $\operatorname{Im} F$-approach [5] and the real time Keldysh technique combined with duality arguments elaborated here.

\section{6. $I-V$ curve and voltage fluctuations}

Now we turn to concrete results. As a first step, let us reconstruct the results [5] for the average voltage $\langle V\rangle$. Making use of Eqs. (43), (44) together with the relation (42) and the expressions for the Green functions (36)-(40) and keeping in mind the detailed balance condition

$$
\mathcal{P}_{X, X^{\prime}}(\omega)=\exp \left(\frac{\omega}{T}\right) \mathcal{P}_{X, X^{\prime}}(-\omega)
$$

we obtain

$$
\langle V\rangle=\frac{\Phi_{0} L v \gamma_{Q P S}^{2}}{2} \varsigma^{2}\left(\frac{\Phi_{0} I}{2}\right) \sinh \left(\frac{\Phi_{0} I}{2 T}\right)
$$

where we introduced

$$
\varsigma(\omega)=\tau_{0}^{\lambda}(2 \pi T)^{\lambda-1} \frac{\Gamma\left(\frac{\lambda}{2}-\frac{i \omega}{2 \pi T}\right) \Gamma\left(\frac{\lambda}{2}+\frac{i \omega}{2 \pi T}\right)}{\Gamma(\lambda)},
$$

$\tau_{0} \sim 1 / \Delta_{0}$ is the QPS core size in time and $\Gamma(x)$ is the Gamma-function. Further assuming that $v \tau_{0} \sim x_{0}$ (where $x_{0} \sim \xi$ is the QPS core size in space) we observe that the result (65) fully matches with that derived in [5] by means of a different technique.

Now let us analyze the general expressions for the voltage noise (45)-(50). At zero bias $I \rightarrow 0$ the term $S_{\Omega}^{a}$ van- ishes, and the equilibrium noise spectrum $S_{\Omega}=S_{\Omega}^{(0)}+S_{\Omega}^{r}$ can be obtained directly from FDT, see also [22]. At nonzero bias values the QPS noise becomes non-equilibrium. In the limit $\Omega \rightarrow 0$ the terms $S_{\Omega}^{(0)}$ and $S_{\Omega}^{r}$ vanish and the voltage noise $S_{\Omega \rightarrow 0} \equiv S_{0}$ is determined solely by $S_{\Omega}^{a}$. Then from Eq. (49) we get

$$
\begin{aligned}
S_{0}= & \Phi_{0}^{2}\left(\Gamma_{Q P S}(I)+\Gamma_{Q P S}(-I)\right)= \\
& =\Phi_{0} \operatorname{coth}\left(\frac{\Phi_{0} I}{2 T}\right)\langle V\rangle,
\end{aligned}
$$

where $\langle V\rangle$ is specified in Eq. (65). Combining the result (67) with Eqs. (65), (66) we obtain

$$
S_{0} \propto \begin{cases}T^{2 \lambda-2}, & T>>\Phi_{0} I \\ I^{2 \lambda-2}, & T<<\Phi_{0} I\end{cases}
$$

At higher temperatures $T>\Phi_{0} I$ (although $T<<\Delta_{0}$ ) Eq. (68) accounts for equilibrium voltage noise $S_{0}=2 T R$ of a linear Ohmic resistor $R=\langle V\rangle / I \propto T^{2 \lambda-3}$ [5]. In the low temperature limit $T<<\Phi_{0} I$ it describes QPS-induced shot noise $S_{0}=\Phi_{0}\langle V\rangle$ obeying Poisson statistics with an effective "charge" equal to the flux quantum $\Phi_{0}$.

The above analysis allows to answer the question about the physical origin of shot noise in superconducting nanowires. We conclude that voltage shot noise is produced by coherent tunneling of magnetic flux quanta $\Phi_{0}$ across the wire. In the dual picture employed here such flux quanta can be viewed as charged quantum particles passing through and being scattered at an effective "tunnel barrier". We also note that the result analogous to Eq. (67) was previously derived for thermally activated phase slips (TAPS) [26].

It is instructive to mention that our analysis also allows to recover higher correlators of the voltage operator (30). Let us define the voltage cumulants

$$
\mathcal{C}_{n}=(-i)^{n} \lim _{t \rightarrow \infty}\left[\frac{1}{t} \partial_{z}^{n} \log \left\langle\exp \left(i z \int_{0}^{t} d t_{1} V\left(t_{1}\right)\right)\right\rangle\right]_{z=0} .
$$

Within the accuracy of our perturbation theory the terms $\mathcal{C}_{k} \propto \gamma_{Q P S}^{2}$ with $k<n$ generated in the right-hand side of Eq. (69) can be ignored. Then $\mathcal{C}_{n}$ coincides with the Fourier transformed correlators (30), i.e., $\mathcal{C}_{2}=S_{0}$ etc. Proceeding perturbatively in $\gamma_{\text {QPS }}$ and employing Eqs. (65), (66), at $T \rightarrow 0$ we obtain [12]

$$
\mathcal{C}_{n}=\Phi_{0}^{n-1}\langle V\rangle=\frac{\pi^{2} v L \gamma_{Q P S}^{2} \tau_{0}^{2 \lambda} \Phi_{0}^{n}}{2^{2 \lambda-2} \Gamma^{2}(\lambda)}\left|\Phi_{0} I\right|^{2 \lambda-2} .
$$

The above results allow to fully describe statistics of QPSinduced voltage fluctuations in superconducting nanowires in the low frequency limit. 
Another interesting situation is that of sufficiently high frequencies and/or long wires $v / L<<\Omega<<\Delta_{0}$. In this limit we find

$$
S_{\Omega}^{(0)}=\frac{\lambda}{8 \pi e^{2}} \frac{\Omega \operatorname{coth}\left(\frac{\Omega}{2 T}\right)}{\left(\Omega / 2 E_{C}\right)^{2}+(\lambda / \pi)^{2}} .
$$

It is easy to observe that this contribution does not depend on the wire length $L$. At low $T$ and $\Omega / \lambda \gtrsim E_{C}=e^{2} / 2 C$ we have $S_{\Omega}^{(0)} \propto 1 / \Omega$, i.e. the wire can generate $1 / f$ voltage noise. Let us now evaluate the QPS terms $S_{\Omega}^{r}$ and $S_{\Omega}^{a}$. In doing so, it is straightforward to demonstrate that the latter term scales linearly with the wire length $L$ whereas the former shows weaker dependence on $L$. Hence, the term $S_{\Omega}^{r}$ can simply be dropped in the long wire limit. For the remaining QPS term $S_{\Omega}^{a}$ we get

$$
\begin{aligned}
S_{\Omega}^{a}= & \frac{L \lambda^{2} v \gamma_{Q P S}^{2}}{4 e^{2}}\left[\varsigma\left(\frac{\Phi_{0} I}{2}-\Omega\right)-\varsigma\left(\frac{\Phi_{0} I}{2}+\Omega\right)\right] \times \\
& \times \frac{\sinh \left(\frac{\Phi_{0} I}{2 T}\right) \varsigma\left(\frac{\Phi_{0} I}{2}\right)}{\left(\left(\Omega / 2 E_{C}\right)^{2}+(\lambda / \pi)^{2}\right) \sinh \left(\frac{\Omega}{2 T}\right)} .
\end{aligned}
$$

At $T \rightarrow 0$ Eq. (72) yields

$$
S_{\Omega}^{a} \propto\left\{\begin{array}{cl}
I^{\lambda-1}\left(I-2 \Omega / \Phi_{0}\right)^{\lambda-1}, & \Omega<\Phi_{0} I / 2, \\
0, & \Omega>\Phi_{0} I / 2 .
\end{array}\right.
$$

In order to interpret this threshold behavior it is necessary to bear in mind that at $T=0$ each QPS event can excite $2 N$ plasmons $(N=1,2 \ldots)$ with total energy $E=\Phi_{0} I$ and total zero momentum. The left and the right moving plasmons (each group carrying total energy $E / 2$ ) eventual-

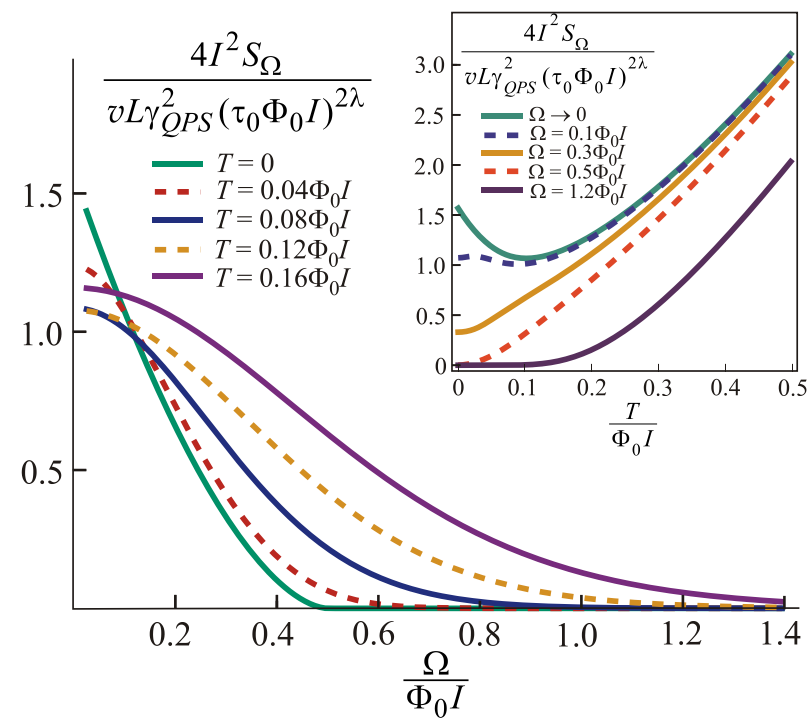

Fig. 5. (Color online) The frequency dependence of the QPS noise spectrum $S_{\Omega}(72)$ at $\lambda=2.7$, large $E_{C}$ and different $T$ in the long wire limit. The inset shows $S_{\Omega}$ as a function of $T$. ly reach respectively the left and the right wire ends. One group gets dissipated at the grounded end of the wire while another one causes voltage fluctuations with frequency $\Omega$ measured by a detector. Clearly, at $T=0$ this process is only possible at $\Omega<E / 2$ in the agreement with Eq. (73).

The result (72) is also illustrated in Fig. 5. At sufficiently small $\Omega$ one observes a non-monotonous dependence of $S_{\Omega}$ on $T$. This behavior is a direct consequence of quantum coherent nature of QPS noise. We also emphasize that at non-zero $T$ Eq. (72) does not coincide with the zero frequency result (67) even in the limit $\Omega \rightarrow 0$. This difference has to do with the order of limits: Before taking the zero frequency limit in Eq. (72) one should formally set $L \rightarrow \infty$. Then one finds

$$
S_{\Omega \rightarrow 0}^{a}(I)=-v L T \gamma_{Q P S}^{2} \Phi_{0}^{2} \varsigma\left(\frac{\Phi_{0} I}{2}\right) \varsigma^{\prime}\left(\frac{\Phi_{0} I}{2}\right) \sinh \left(\frac{\Phi_{0} I}{2 T}\right)
$$

Comparing Eqs. (74) and Eq. (67) (in the latter equation the limit $\Omega \rightarrow 0$ was taken prior to sending the wire length $L$ to infinity) one observes the identity

$$
S_{0}(I, T)-S_{\Omega \rightarrow 0}^{a}(I, T)=2 T R(I, T),
$$

implying that both expressions (74) and (67) coincide only at $T=0$, while at any non-zero $T$ the noise power $S_{0}(I, T)$ (67) exceeds one in Eq. (74) and grows monotonously with temperature.

The above analysis is merely applicable to sufficiently long wires in which case the main dissipation mechanism is due Mooij-Schön plasmons [24] propagating along the wire and carrying energy out of the system. One can also consider the limit of shorter wires where such plasmons are irrelevant and other dissipation mechanisms come into play. In such wires one typically has $L<<v / T$, i.e., the system spatial dimension is much shorter than that in time direction. Under such conditions it is convenient to split our analysis into two parts and consider the effect of high frequency modes (short scales) and low frequency ones separately. This procedure was already described elsewhere $[22,27]$ and is known as the so-called two stage scaling.

High frequency modes can be accounted for by means of the well known Berezinskii-Kosterlitz-Thouless (BKT) renormalization group (RG) approach. The corresponding RG equations read

$$
\frac{d \zeta}{d \ln \Lambda}=(2-\lambda) \zeta, \quad \frac{d \lambda}{d \ln \Lambda}=-32 \pi^{2} \zeta^{2} \lambda^{2} K(\lambda),
$$

where $\zeta=\gamma_{Q P S} \Lambda^{2}$ is the dimensionless coupling parameter, $\Lambda$ is the renormalization scale and $K(\lambda)$ is some nonuniversal function (which depends on the renormalization scheme) equal to one at the quantum BKT phase transition point $\lambda=2$ which separates a superconducting (ordered) phase $\lambda>2$ with bound QPS-antiQPS pairs and a disordered phase $\lambda<2$ with unbound QPS [5]. 
As usually, we start renormalization at the shortest scale $\Lambda \sim \xi_{c}=\sqrt{\xi^{2}+v^{2} / \Delta^{2}}$ and proceed to bigger scales. As above, for simplicity we set $\xi_{c} \sim \xi$. Within the first order perturbation theory in $\zeta$ one can ignore weak renormalization of the parameter $\lambda$. With this accuracy the solution of Eqs. (76) takes the form $\gamma_{Q P S}(\Lambda)=\gamma_{Q P S}(\xi / \Lambda)^{\lambda}$. Terminating this RG procedure at the maximum scale $\Lambda \sim L$ we arrive at the renormalized QPS amplitude for our system

$$
\tilde{\gamma}_{Q P S}=\gamma_{Q P S}(\xi / L)^{\lambda} \text {. }
$$

This equation demonstrates that interaction-induced renormalization of the QPS amplitude is usually quite important. This effect can be disregarded only for very small values of $\lambda<<1 / \ln (L / \xi)$ which is not the case here.

At all time scales exceeding $L / v$ the system behaves as effectively zero-dimensional one characterized by the QPS amplitude (77). Repeating the whole analysis of voltage fluctuations we again arrive at the general results for the voltage-voltage correlator in the form (45)-(50), with all the Green functions being independent of the spatial coordinates. This general result can be rewritten as

$$
\begin{gathered}
S_{\Omega}=S_{\Omega}^{(0)}-\frac{\tilde{\gamma}_{Q P S}^{2} L^{2} \Omega^{2} \operatorname{coth}\left(\frac{\Omega}{2 T}\right)}{16 e^{2}} G_{\varphi \chi}^{R}(\Omega) G_{\varphi \chi}^{R}(\Omega) \times \\
\times\left(P\left(\Omega+\Phi_{0} I\right)+P\left(\Omega-\Phi_{0} I\right)-\bar{P}\left(-\Omega-\Phi_{0} I\right)-\bar{P}\left(-\Omega+\Phi_{0} I\right)-\right. \\
\left.-P\left(\Phi_{0} I\right)-P\left(-\Phi_{0} I\right)+\bar{P}\left(\Phi_{0} I\right)+\bar{P}\left(-\Phi_{0} I\right)\right)+ \\
\Omega^{2}\left(\operatorname{coth}\left(\frac{\Omega+\Phi_{0} I}{2 T}\right)-\operatorname{coth}\left(\frac{\Omega}{2 T}\right)\right) \\
\frac{16 e^{2}}{2} G_{\varphi \chi}^{R}(\Omega) G_{\varphi \chi}^{R}(-\Omega) \times \\
\times\left(P\left(\Omega+\Phi_{0} I\right)+\bar{P}\left(\Omega+\Phi_{0} I\right)-P\left(-\Omega-\Phi_{0} I\right)-\right. \\
\left.-\bar{P}\left(-\Omega-\Phi_{0} I\right)\right)+(\Omega \rightarrow-\Omega),
\end{gathered}
$$

where

$$
P(\omega)=\int_{0}^{\infty} d t e^{i \omega t} \mathrm{e}^{i G_{\chi \chi}^{K}(t)-G_{\chi \chi}^{K}(0)+\frac{i}{2} G_{\chi \chi}^{R}(t)},
$$

and the Green functions are equal to

$$
\begin{gathered}
G_{\varphi \varphi}^{R}(\omega)=\frac{2 \pi}{i \mu \omega\left(1-i \omega \tau_{R C}\right)-\frac{2 \lambda v}{L}}, \\
G_{\varphi \chi}^{R}(\omega)=-\frac{4 \pi i \lambda v}{L(\omega+i 0)\left(i \mu \omega\left(1-i \omega \tau_{R C}\right)-\frac{2 \lambda v}{L}\right)},
\end{gathered}
$$

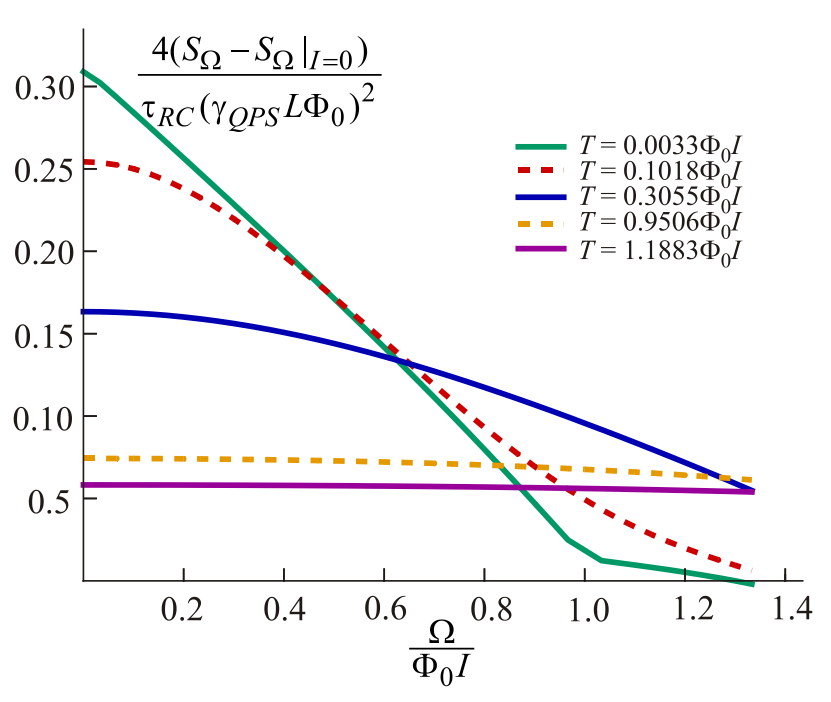

Fig. 6. The frequency dependence of the shot noise spectrum $S_{\Omega}-\left.S_{\Omega}\right|_{I=0}$ at $\mu=1.025, \Phi_{0} I \tau_{R C}=0.3, \frac{2 \lambda \nu}{\mu L}=3.33 \Phi_{0} I$, and different $T$ in the short wire limit.

$$
G_{\chi \chi}^{R}(\omega)=\frac{4 \pi i \mu \lambda v\left(1-i \omega \tau_{R C}\right)}{L(\omega+i 0)\left(i \mu \omega\left(1-i \omega \tau_{R C}\right)-\frac{2 \lambda v}{L}\right)} .
$$

Here $\tau_{R C}=R_{S} C$ is the RC-time and $\mu=R_{Q} / R_{S}$ is the shunt dimensionless conductance. One can further simplify the above expression provided all relevant energy scales, such as $\Omega, \Phi_{0} I$ and $T$ remain smaller than both $1 / \tau_{R C}$ and $\lambda v /(\mu L)$. In that case $\omega G_{\varphi \chi}^{R}(\omega) \approx 2 \pi i$ is approximately constant and

$$
\begin{aligned}
S_{\Omega} & \approx \frac{1}{4} \Phi_{0}^{2} \tilde{\gamma}_{Q P S}^{2} L^{2} \operatorname{coth}\left(\frac{\Omega+\Phi_{0} I}{2 T}\right)\left(P\left(\Omega+\Phi_{0} I\right)+\right. \\
+ & \left.\bar{P}\left(\Omega+\Phi_{0} I\right)-P\left(-\Omega-\Phi_{0} I\right)-\bar{P}\left(-\Omega-\Phi_{0} I\right)\right)+ \\
& +\frac{1}{4} \Phi_{0}^{2} \tilde{\gamma}_{Q P S}^{2} L^{2} \operatorname{coth}\left(\frac{\Omega-\Phi_{0} I}{2 T}\right)\left(P\left(\Omega-\Phi_{0} I\right)+\right. \\
& \left.+\bar{P}\left(\Omega-\Phi_{0} I\right)-P\left(-\Omega+\Phi_{0} I\right)-\bar{P}\left(-\Omega+\Phi_{0} I\right)\right) .
\end{aligned}
$$

The frequency dependence of QPS-induced shot noise power spectrum in the short wire limit is illustrated in Fig. 6.

\section{Comparison with four-probe measurement scheme}

Let us now consider another system configuration displayed in Fig. 2. We will again stick to the wire Hamiltonian $\hat{H}_{\text {wire }}$ in its dual representation defined by Eqs. (26), (19) and (25), where, as before, canonically conjugate flux and charge operators $\hat{\Phi}(x)$ and $\hat{\chi}(x)$ obey the commutation relation (15). The effect of an external current bias is now accounted for within Eq. (19) by means of the shift of the flux operator $\hat{\Phi}(x) \rightarrow \hat{\Phi}(x)+\mathcal{L}_{\text {kin }} I$. The phase difference 
between the two wire points $x_{1}$ and $x_{2}$ can then be defined as

$$
\hat{\varphi}\left(x_{1}\right)-\hat{\varphi}\left(x_{2}\right)=2 e \int_{x_{2}}^{x_{1}} d x \hat{\Phi}(x)
$$

Employing the Josephson relation one easily recovers the operator for the voltage difference between the points $x_{1}$ and $x_{2}$ in the form:

$$
\hat{V}=\frac{1}{\Phi_{0} C_{w}}\left(\nabla \hat{\chi}\left(x_{1}\right)-\nabla \hat{\chi}\left(x_{2}\right)\right) .
$$

The above expressions allow to directly evaluate voltage correlators perturbatively in $\gamma_{Q P S}$. In the case of the four-point measurement scheme of Fig. 2 the calculation is similar to one already carried out above for the two-point measurements. Therefore we can directly proceed to our final results. Evaluating the first moment of the voltage operator $\langle\hat{V}\rangle$ we again reproduce Eqs. (43), (65). For the voltage noise power spectrum $S_{\Omega}$ we now obtain

$$
S_{\Omega}=\int d t \mathrm{e}^{i \Omega t}\langle V(t) V(0)\rangle=S_{\Omega}^{(0)}+S_{\Omega}^{Q P S},
$$

where the term $S_{\Omega}^{(0)}$ describes equilibrium voltage noise in the absence of QPS (71) and [13]

$$
\begin{gathered}
S_{\Omega}^{Q P S}=\frac{\gamma_{Q P S}^{2} e^{2} \operatorname{coth}\left(\frac{\Omega}{2 T}\right)}{4 \pi^{2} C_{w}^{2}} \int \frac{d k}{2 \pi} \mathcal{M}_{k}(\Omega)\left(P_{k}\left(\Phi_{0} I\right)-\right. \\
\left.-\bar{P}_{k}\left(-\Phi_{0} I\right)+P_{k}\left(-\Phi_{0} I\right)-\bar{P}_{k}\left(\Phi_{0} I\right)\right)+ \\
+\frac{\gamma_{Q P S}^{2} e^{2} \operatorname{coth}\left(\frac{\Omega}{2 T}\right)}{4 \pi^{2} C_{w}^{2}} \int \frac{d k}{2 \pi} \mathcal{S}_{k}(\Omega) \mathcal{S}_{-k}(\Omega)\left(\bar{P}_{k}\left(-\Omega-\Phi_{0} I\right)-\right. \\
\left.-P_{k}\left(\Omega+\Phi_{0} I\right)+\bar{P}_{k}\left(-\Omega+\Phi_{0} I\right)-P_{k}\left(-\Omega-\Phi_{0} I\right)\right)+ \\
+\frac{\gamma_{Q P S}^{2} e^{2}\left(\operatorname{coth}\left(\frac{\Omega+\pi I / e}{2 T}\right)-\operatorname{coth}\left(\frac{\Omega}{2 T}\right)\right)}{4 \pi^{2} C_{w}^{2}} \int \frac{d k}{2 \pi} \mathcal{S}_{k}(\Omega) \times \\
\quad \times \mathcal{S}_{-k}(-\Omega)\left(P_{k}\left(\Omega+\Phi_{0} I\right)+\bar{P}_{k}\left(\Omega+\Phi_{0} I\right)-\right. \\
\left.-P_{k}\left(-\Omega-\Phi_{0} I\right)-\bar{P}_{k}\left(-\Omega-\Phi_{0} I\right)\right)+(\Omega \rightarrow-\Omega)(87)
\end{gathered}
$$

is the voltage noise power spectrum generated by quantum phase slips. Eq. (87) contains the function

$P_{k}(\omega)=\int d x \mathrm{e}^{i k x} \int_{0}^{\infty} d t \mathrm{e}^{i \omega t} \mathrm{e}^{i G_{\chi \chi}^{K}(x, t)-i G_{\chi \chi}^{K}(0,0)+\frac{i}{2} G_{\chi \chi}^{R}(x, t)}$

and geometric form-factors $\mathcal{M}_{k}(\Omega)$ and $\mathcal{S}_{k}(\Omega)$ which explicitly depend on $x_{1}$ and $x_{2}$. E.g., setting $x_{1}=L / 2$ and $x_{2}=-L / 2$ we obtain

$$
\begin{gathered}
\mathcal{M}_{k}(\Omega)=(4 \pi \lambda)^{2} \mathrm{e}^{\frac{i \Omega L}{v}} \frac{\sin \left(\frac{k L}{2}\right)}{v k}\left(\frac{2 \sin \left(\frac{k L}{2}\right)}{v k}+\right. \\
\left.+\frac{\sin \left(\frac{(2 \Omega+v k) L}{2 v}\right)}{2 \Omega+v k}+\frac{\sin \left(\frac{(2 \Omega-v k) L}{2 v}\right)}{2 \Omega-v k}\right), \\
\mathcal{S}_{k}(\Omega)=4 \pi \lambda \mathrm{e}^{\frac{i \Omega L}{2 v}\left(\frac{\sin \left(\frac{(\Omega+v k) L}{2 v}\right)}{\Omega+v k}+\frac{\sin \left(\frac{(\Omega-v k) L}{2 v}\right)}{\Omega-v k}\right) .} .
\end{gathered}
$$

These form-factors oscillate as functions of $\Omega$ due to the interference effect at the boundaries of a thinner wire segment. Such oscillations make the result for the shot noise in general substantially different as compared to that evaluated for the setup of Fig. 1. For $\Omega>>/ L$ one has

$$
\begin{gathered}
\mathcal{M}_{k}(\Omega) \approx \pi L \frac{(4 \pi \lambda)^{2}}{v^{2}} \mathrm{e}^{\frac{i \Omega L}{v}} \delta(k), \\
\mathcal{S}_{k}(\Omega) \mathcal{S}_{-k}(\Omega) \approx \frac{(4 \pi \lambda)^{2}}{v^{2}} \mathrm{e}^{\frac{i \Omega L}{v}}\left(\frac{\pi L}{2} \delta\left(k+\frac{\Omega}{v}\right)+\right. \\
\left.+\frac{\pi L}{2} \delta\left(k-\frac{\Omega}{v}\right)\right), \\
\mathcal{S}_{k}(\Omega) \mathcal{S}_{-k}(-\Omega) \approx \frac{(4 \pi \lambda)^{2}}{v^{2}}\left(\frac{\pi L}{2} \delta\left(k+\frac{\Omega}{v}\right)+\right. \\
\left.+\frac{\pi L}{2} \delta\left(k-\frac{\Omega}{v}\right)\right) .
\end{gathered}
$$

Neglecting the contributions (91) and (92) containing fast oscillating factors $\mathrm{e}^{\frac{i \Omega L}{v}}$ and combining the remaining term (93) with Eq. (87), we obtain

$$
S_{\Omega}^{Q P S}=S_{\Omega}^{a} / 2,
$$

where $S_{\Omega}^{a}$ is defined in Eq. (72). This result implies that shot noise measured by each of our two detectors in the configuration of Fig. 2 is 4 times smaller than that detected with the aid of the setup of Fig. 1. The result (94) is also illustrated in Fig. 7.

At $T \rightarrow 0$ Eq. (94) obviously yields the same threshold behavior (73). The physical reasons for this behavior are the same as before, one should just bear in mind that in the long wire limit and for non-zero $\Omega$ the two groups of plasmons — left moving and right moving ones — each carrying the energy $E / 2$ become totally uncorrelated implying that at $T=0$ voltage noise can only be detected at $\Omega<E / 2$ in the agreement with Eq. (73). 


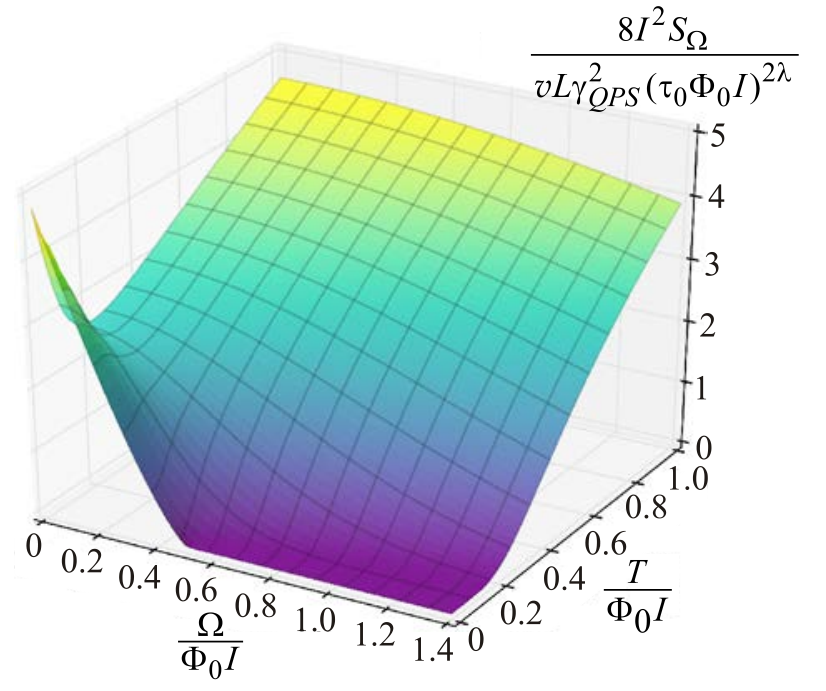

Fig. 7. (Color online) The dependence of QPS noise power $S_{\Omega}$ (72) on frequency $\Omega$ and temperature $T$ at $\lambda=2.3$.

\section{Concluding remarks}

In this paper we combined the phase-charge duality arguments with Keldysh path integral technique and demonstrated that quantum phase slips may cause voltage fluctuations in superconducting nanowires. In the presence of a current bias $I$ quantum tunneling of the magnetic flux quanta $\Phi_{0}$ across the wire yields. Poissonian statistics of such fluctuations. In both limits of longer and shorter nanowires shot noise exhibits a non-trivial power law dependence of its spectrum $S_{\Omega}$ on temperature $T$, external bias $I$ and frequency $\Omega$. We also demonstrated that in the zero temperature limit $S_{\Omega}$ decreases with increasing frequency and vanishes beyond a threshold value of $\Omega$. At low enough frequencies $S_{\Omega}$ may depend non-monotonously on temperature due to quantum coherent nature of QPS noise.

It is important to emphasize that the perturbative in $\gamma_{\text {QPS }}$ approach employed here is fully justified in the socalled "superconducting" phase, i.e. for longer wires with $\lambda>2$ [5] and for shorter wires at $R_{s}<R_{Q}$ [14]. In the "non-superconducting" regime, i.e. for wires either with $\lambda<2$ or with $R_{S}>R_{Q}$ the QPS amplitude gets effectively renormalized to higher values and, hence, the perturbation theory eventually turns obsolete. Nevertheless, even in this case our predictions may still remain applicable at high enough temperature, frequency and/or current values. In the opposite low energy limit long wires with $\lambda<2$ show an insulating behavior, as follows from the exact solution of the corresponding sine-Gordon model [28]. The same is true also for shorter wires at low energies and $R_{S} \rightarrow \infty$. This behavior suggests that also voltage fluctuations become large in this limit.

Finally, we would like to point out that voltage fluctuations detected in superconducting nanowires may in general depend on the particular measurement setup. This dependence can be important and should be observed while performing noise measurements in such nanowires. In addition, the results of our theoretical analysis need to be taken into account while optimizing the operation of QPS qubits [29].

This work was supported in part by RFBR grant No. 15-02-08273.

1. K.Yu. Arutyunov, D.S. Golubev, and A.D. Zaikin, Phys. Rep. 464, 1 (2008).

2. A. Bezryadin, J. Phys.: Condens. Matter 20, 043202 (2008).

3. A.D. Zaikin, in: Handbook of Nanophysics: Nanotubes and Nanowires, CRC Press, Boca Raton, FL (2010), p. 40.

4. A. Bezryadin, Superconductivity in Nanowires, Wiley-VCH, Weinheim (2013).

5. A.D. Zaikin, D.S. Golubev, A. van Otterlo, and G.T. Zimanyi, Phys. Rev. Lett. 78, 1552 (1997).

6. D.S. Golubev and A.D. Zaikin, Phys. Rev. B 64, 014504 (2001).

7. A. Bezryadin, C.N. Lau, and M. Tinkham, Nature 404, 971 (2000).

8. C.N. Lau, N. Markovic, M. Bockrath, A. Bezryadin, and M. Tinkham, Phys. Rev. Lett. 87, 217003 (2001).

9. M. Zgirski, K.P. Riikonen, V. Touboltsev, and K.Y. Arutyunov, Phys. Rev. B 77, 054508 (2008).

10. X.D.A. Baumans et al., Nat. Commun. 7, 10560 (2016).

11. A.G. Semenov and A.D. Zaikin, Phys. Rev. B 94, 014512 (2016).

12. A.G. Semenov and A.D. Zaikin, Fortschr. Phys. 64, 1600043 (2017).

13. A.G. Semenov and A.D. Zaikin, J. Supercond. Nov. Magn. 30, 139 (2017).

14. G. Schön and A.D. Zaikin, Phys. Rep. 198, 237 (1990).

15. A. van Otterlo, D.S. Golubev, A.D. Zaikin, and G. Blatter, Eur. Phys. J. B 10, 131 (1999).

16. S.V. Panyukov and A.D. Zaikin, J. Low Temp. Phys. 73, 1 (1988).

17. D.V. Averin and A.A. Odintsov, Phys. Lett. A 140, 251 (1989).

18. A.D. Zaikin, J. Low Temp. Phys. 80, 223 (1990).

19. J.E. Mooij and Yu.V. Nazarov, Nat. Phys. 2, 169 (2006).

20. A.G. Semenov, PhD Thesis, P.N. Lebedev Physics Institute, Moscow (2010).

21. A.M. Hriscu and Yu.V. Nazarov, Phys. Rev. B 83, 174511 (2011).

22. A.G. Semenov and A.D. Zaikin, Phys. Rev. B 88, 054505 (2013).

23. A.V. Galaktionov, D.S. Golubev, and A.D. Zaikin, Phys. Rev. B 68, 235333 (2003).

24. J.E. Mooij and G. Schön, Phys. Rev. Lett. 55, 114 (1985).

25. U. Weiss, Quantum Dissipative Systems, World Scientific, Singapore (2008).

26. D.S. Golubev and A.D. Zaikin, Phys. Rev. B 78, 144502 (2008).

27. A.D. Zaikin, D.S. Golubev, A. van Otterlo, and G.T. Zimanyi, Usp. Fiz. Nauk 168, 244 (1998) [Phys. Usp. 42, 226 (1998)].

28. D. Controzzi, F.H.L. Essler, and A.M. Tsvelik, Phys. Rev. Lett. 86, 680 (2001) and References therein.

29. J.E. Mooij and C.J.P.M. Harmans, New J. Phys. 7, 219 (2005) 